\title{
A Markov Model Accounting for Charge Recovery in Energy Harvesting Devices
}

\author{
Leonardo Badia, Elisa Feltre, and Elvina Gindullina \\ University of Padova, Dept. of Information Engineering, via Gradenigo 6B, 35131 Padova, Italy \\ email: \{badia,elisa.feltre,elvina.gindullina\}@dei.unipd.it
}

\begin{abstract}
Energy harvesting is an important feature that can be implemented in mobile devices to provide them with extended autonomy, yet it poses several challenges in terms of optimal battery usage. In this paper, we highlight that some non-ideal effects, especially the so-called "charge recovery," can have a dramatic impact on the operation policy of autonomous devices. To do so, we construct a Markov model, where we introduce a bidimensional battery value, including the apparent energy level, which is what available at the electrodes to power the device, and the actual energy level stored in the battery. When power is not drained by the device, these levels tend to equalize due to charge recovery, but, in the case of intense battery usage, they can be significantly different. We show that this non-ideality leads to considerably different estimates of undesirable events such as battery outages, and may cause a general underutilization of the devices if not properly accounted for.
\end{abstract}

Index Terms-Battery management; wireless sensor networks; green design; renewable energy sources; stochastic processes.

\section{INTRODUCTION}

$\mathbf{F}$ UTURE wireless networks are deemed to reach a pervasive penetration in many technological scenarios of everyday's life. One major downside of this achievement is an unprecedented increase in worldwide energy consumption: it is estimated that power demands of the ICT ecosystem are approaching one tenth of the electricity generation on the planet [1]. In spite of their limited energy requirements for individual tasks, their intensive usage makes tablets and smartphones more energy-hungry on a long time scale than domestic electric appliances like the refrigerator; furthermore, the latter is generally shared in each household, while the formers are individually owned. These estimates can become even more worrisome if embodied energy is also included [2].

At the same time, one of the main limitations putting the brakes on further developments of applications for wireless networks is the limited autonomy of present-day batteries [3]. In principle, mobile nodes should be able to exploit both wireless connectivity and sufficiently long energetic autonomy. However, the energy reserve that can be stored in a battery is limited, and even more so for tiny devices.

In light of this, the use of renewable sources integrated in mobile terminals through some energy harvesting techniques is gaining appeal as a way to reduce global energy consumption, with the benefits of controlling pollution and greenhouse emissions, as well as enabling longer operation for wireless

This work has received funding from the European Unions Horizon 2020 research and innovation programme under the Marie Skłodowska-Curie grant agreement No. 675891 (SCAVENGE). communications [4]. However, renewable sources are too erratic to guarantee reliable functioning, thus batteries are still needed to provide backup energy supply when the devices are not powered by the harvesting process. Energetic sustainability requires the combination of the harvesting process with a smart management of the battery seen as an energy buffer [5]. This is needed to both store the energy harvested from the environment for a later use, and also to guarantee quality objectives, while still dealing with the limited energy supply.

In many existing contributions [3], [6], mathematical models for energy harvesting devices are proposed, often with a focus on designing power management policies. A single device can be treated as performing specific energy-consuming tasks (e.g., sending data and/or sensing the environment), while being powered by a renewable source. Without loss of generality, we will see the tasks as the transmission of packets, generated according to a known arrival process to the data queue of the device, located in its transmission buffer. Instead, energy is harvested and stored in the device battery, seen as an energy quеие. Thus, we a double queueing system with joint service, meaning that a packet can be transmitted if and only if the battery has got enough energy. Data packets and energy are simultaneously taken from the transmission buffer and the battery, respectively; if either queue (data or energy) is empty, packet transmission has to wait for replenishment.

The basic model outlined above dictates that the energy level of the battery only changes when a transmission occurs. This contrasts with the practical observation that batteries are subject to non-idealities. An example is leakage [7], causing the battery level to decrease even in the absence of transmissions. An even more complicated phenomenon is the so-called charge recovery effect [8], [9], according to which the battery level apparently raises when no energy is drained.

The contribution of this paper is to explore a more realistic battery model, where these non-idealities are included. We implement a discrete-time finite-state Markov chain, and investigate the battery behavior as a function of the characterizing parameters, especially the probabilities of deep discharge and battery recovery. To do so, we extend the standard representation considering the lengths of the data and the energy queue into a triple of values, in which the energy level is split into the real and the apparent one. This duality is key for charge recovery, which is actually just an increase of the apparent battery level. The model is solved and results are discussed, in particular for the probability of energy outage, i.e., that the battery cannot power the device anymore. Actually, we need distinguish between the real and apparent outage; such 
a difference implies that the occurrence of the outage event can be severely overestimated, and we quantitatively show how the parameters of charge recovery effect impact on this.

The rest of this paper is organized as follows. In Section II we discuss analytical models proposed in the literature for mobile batteries and their non-ideal effects. In Section III we outline our contribution of a Markov model where the battery recovery effect is taken into account by expanding the state of the double queue into a triple of values. The model solution is discussed in Section IV, while Section V presents numerical results, showing that neglecting battery recovery effects can lead to overestimating of the apparent outage probability (and conversely, the battery is almost never depleted for real). Finally, we draw the conclusions in Section VI.

\section{BACKGROUND ON BATTERY NON-IDEALITIES}

Batteries employed for mobile devices usually include multiple cells, each containing two electrodes separated by an electrolyte, referred to as the active materials. The electrolyte participates in the electrochemical reaction leading to charging and discharging of the battery [10]. The cell is discharged when it is connected to an external circuit and an oxidereduction reaction transfers charge bearers among the electrodes, thus converting chemical into electrical energy. This process goes on until the battery reaches a cutoff voltage, i.e., the one at which it can be considered as disconnected. Alternatively, the discharge can be reversed by supplying the battery with electrical energy that is converted back into chemical energy stored by the active materials.

Yet, this entire process is far from ideal, depending on factors such as the environment temperature and/or the depth of charge-and-discharge cycles. For these reason, a battery can hardly be treated as an ideal source if certain conditions are not met. For example, [11] describes several non-idealities, such as the variation of the internal resistance depending on the external temperature. Also, capacity fading effects may take place, causing a degradation of the maximum amount of energy that can be stored in the battery, primarily depending on how many charge/discharge cycles have been performed, as well as their depth [12]. Even when the battery is inactive, inner electrochemical processes still take place, which can cause a constant leakage [13]. All these aspects are heavily influenced by the kind of battery, its weariness, and environmental conditions (such as the external temperature); a detailed analysis of the impact that these limitations is available in [14].

In this paper, we focus instead on a previously overlooked aspect, the charge recovery effect [15], which corresponds to the rise in the apparent energy level of a battery that is not discharged for a while. The reason is as follows. When the cells are fully charged, active materials have maximum concentration in the entire cell; when a discharging current is generated, those close to the cathode are consumed by electrochemical reactions, and replaced by other active materials moving towards the electrode according to a diffusion mechanism. If the current intensity is above a limiting value, this mechanism is not able to compensate the consumption of active materials around the electrode, which lowers the voltage. Especially, if the cutoff voltage is reached, electrochemical reactions halt and the battery is seen as discharged, even though some charge is still present in the cell. Now that the active materials are no longer drained, though, they diffuse across the cell and their concentration around the electrode increases. A charge recovery effect can be observed as causing a slow gradual rise of the apparent energy level. The extent of the recovery depends on the duration and the depth of the previous discharge, even though, according to [9], a long and intense discharge impulse increases the inner temperature of the battery, which in turn decreases the recovery effect. This means that if the battery is being constantly discharged, no recovery is possible and the device is no longer powered quite early, so that only a fraction of the battery charge is actually used, in some cases even less than $30 \%$ [15].

A better idea is to cyclically alternate discharge intervals with inactive periods, during which charge recovery is exploited. Setting a duty cycle involves a tradeoff between intense battery usage for a limited period of time, and extended operation time by exploitation of the recovery effect, at the price of not always using the battery, even when needed [8]. Also, note that the recovery effect cannot be exploited indefinitely, since when a new equilibrium of the active materials is reached, the recovery ceases to bring any benefit.

\section{MARKOV MOdEL SETUP}

We formulate a Markov model for energy harvesting devices including the non-idealities such as leakage and charge recovery effects. We refer to a system consisting of a generic wireless transceiver, e.g., a sensor node, transmitting data packets, and the battery powering it, which in turn can be recharged by some energy harvesting mechanism.

As per existing analytical studies [12], the entire system is framed as a double queue with simultaneous service. The general idea is to consider a data queue, describing arrival, buffering, and transmission of traffic, and an energy quеие that includes energy generation, storage, and consumption. For the ease of modeling, both data and energy are discretized into identical atomic units, so that the queues can be seen as having customers arriving and being served one at a time. The clients of the data queue are data packets, all assumed to be of identical size, while we label the one of the energy queue as energy quanta, as discussed in [3]. A quantum is taken as the precise amount of energy that the transceiver requires to transmit one data packet. This in turn means that both queues have joint service of clients, i.e., a data packet can be transmitted only if an energy quantum is spent.

For the sake of simplicity, in line with existing contributions, we consider a discrete time, and packet and energy quantum generations according to independent homogeneous Bernoulli processes of rate $\lambda$ and $\eta$, respectively. This means that, at every time slot, each queue can receive either one new client with probability $\lambda$ (or $\eta$ ), or nothing with probability $1-\lambda$ (or $1-\eta)$. We remark that these assumptions are not critical for the realism of the model, since the time discretization can be made arbitrarily small. Including correlation in client arrivals at both queues may deserve further investigations, since it is 
reasonable to assume that in reality data and energy generation processes have memory (in some cases even considerably so). However, we already studied correlation of arrivals in similar scenarios [12], [16] and therefore we expect the same conclusions to apply here. To keep this analysis simpler, we leave these considerations for future investigations.

This system can be studied with stochastic analysis, characterizing its state as a pair of integers (i.e., the lengths of the both queues). The novelty of the present paper is to consider the system state as a triple of values, so as to keep into account charge recovery effects. Our system state $(q, e, a)$ consists of:

- the number of packets in the data queue, $q$

- the true number of quanta in the energy queue, $e$

- and finally, the apparent energy level, denoted as $a$.

We set values $Q$ and $E$ as the maximum ranges for these non-negative variables. Specifically, $Q$ is the maximum queue length, which relates to the data buffer size, so that $0 \leq q \leq Q$. Instead, $E$ is the maximum value for both the true and the apparent energy levels, $e$ and $a$. We also set the additional constraint that the apparent energy level cannot exceed the true level, so that $0 \leq a \leq e \leq E$. These state variables evolve over a discrete time (for notational simplicity, we omit the temporal reference). We assume that data and energy arrivals, as well as transmissions, take place simultaneously within the time slot. This means that a packet or a quantum of energy arriving at time instant $t$ cannot be immediately exploited but are available for transmission or use from time $t+1$ onwards.

In some contributions [3], the service rate $\mu$ of these queues follows from an operation policy keeping into account the state of the device; in principle, we could denote it as $\mu(q, a){ }^{1}$ This policy can be optimized to pursue an objective, such as maximizing battery lifetime [12]. For the sake of simplicity, we consider instead a constant value of $\mu$, where the only limitations to service are given by not having either packets to transmit or energy quanta to spend, or both. The higher $\mu$, the more intense the battery usage; thus, $\mu \rightarrow 1$ corresponds to what in [3] is referred to as an "aggressive" operation policy.

Importantly, the choice of a fixed $\mu$, instead of an optimized one, is not restrictive for what concerns the conclusion that we will draw later. Indeed, as will be clear from the results, our main message is that most of the outages are apparent, and there is still energy in the battery that is not exploited when the device stops operating. We expect that this conclusion is even more relevant when the discharge rate $\mu$ is optimized, since in that case the optimization is in reality based on the apparent value, and not the real one, as assumed by [3]. The main implication of our analysis will therefore still be true; this could be an interesting subject to explore as a future work.

Another deviation from classic queueing models, which are generally framing the energy queue as a birth-and-death process, is that our battery non-ideality implies that the discharge can sometimes seem to be stronger than one energy quantum. Indeed, an uneven distribution of the active materials leads to an apparent discharge that is heavier than normal. A packet transmission corresponds to the consumption of one energy quantum, therefore we should decrease both $e$ and

${ }^{1}$ We can adapt to the observable values $q$ and $a$, but not to $e$ that is hidden. $a$ by one unit. However, similar to [15], we consider that, whenever a packet is transmitted, a deep discharge event may happen, corresponding to decreasing the true energy level by 1 , same as the normal case, whereas the apparent level decreases by 2 . This way, $a$ can be at times lower than $e$; we model it by defining a deep discharge probability $\alpha$, i.e., whenever a packet is transmitted, a deep discharge happens with conditional probability $\alpha$, whereas a normal discharge (both $e$ and $a$ decrease by 1 ) takes place otherwise.

Some limitations are introduced for the sake of realism. First, discharges (either normal or deep) can only happen when $a>0$. Moreover, the gap between $a$ and $e$ cannot be too high; to discard situations where the distributions of active materials in the cell would be unrealistically uneven, e.g., a very high $e$ and a very low $a$, we set a maximum gap $\Delta$, such that $[e-\Delta]^{+} \leq a \leq e$, where $[x]^{+}=\max (x, 0)$. Thus, deep discharge can only happen if $e-a<\Delta$.

We also consider leakage and especially charge recovery. The former is represented through parameter $\gamma$, i.e., the probability of decreasing the energy levels (both apparent and true) by 1 during a time slot, due to internal chemical degradation of the active materials. Conversely, we describe recovery effects through the probability $\beta$ that, if $a<e$, the apparent energy level is increased by 1 . Similarly to what assumed for the deep discharge, we have to account for the physical nature of the battery, as discussed in Section II; especially, if $e=E$, which means that the battery is fully charged, the distribution of active material will reach a steady state quite soon. This comes from the fact that the intensity of charge recovery depends on a number of internal reactions [17], and bigger amount of active materials causes its faster distribution. For the same reason, in the literature the diffusion coefficient is considered as a function of the state of charge of a battery [18]. Therefore, to accelerate the recovery process if $a<e=E$ we assume that charge recovery of one energy quantum happens with probability $1-\gamma($ instead of $\beta)$. This forces a fully charged battery to recover quickly to the apparent level of maximum charge $a=E$ with probability $1-\gamma$ (i.e., if no leakage happens in the meantime with probability $\gamma$ ). In other words, we exclude the situation of non transition from one energy state to another if no transmission happens.

Finally, for physical coherency, we assume that leakage and recovery cannot happen in the same time slot, thus $\beta+\gamma \leq 1$. For the sake of simplicity, we consider that all these events (deep discharge, charge recovery, leakage) as well as arrivals and services, are independent of each other and identically distributed over time. The investigation of more complex scenarios involving some correlation (e.g., a dependence on an external parameter) is left for future work.

Taking all the constraints into account, we see that the model must include the combination of all choices for $q$ (that has $Q+1$ possible values from 0 to $Q$ ), and the pair $(a, e)$ that instead has $[(E+1)(E+2)-\Delta(\Delta+1)] / 2$ values. Thus, the total number $N$ of states in the Markov chain is

$$
N=(Q+1) \frac{E^{2}+3 E-\Delta^{2}-\Delta+2}{2} .
$$

The Markov chain evolves through changes of its state variables $q, e$, and $a$. This can be represented via balance 
TABLE I

TRANSITIONS OF THE MARKOV CHAIN FROM $(q, e, a)$, with $a>0$

\begin{tabular}{|l|l|l|}
\hline \multicolumn{3}{|c|}{ NO PACKET ARRIVAL, NO SERVICE } \\
\hline \hline$\rightarrow$ new state & probability & explanation \\
\hline$(q, e, a)$ & $(1-\lambda)(1-\eta)(1-\mu)(1-\beta-\gamma)$ & no energy variation \\
\hline$(q, e, \min (e, a+1))$ & $\beta(1-\lambda)(1-\eta)(1-\mu)$ & charge recovery \\
\hline$\left(q,[e-1]^{+},[a-1]^{+}\right)$ & $\gamma(1-\lambda)(1-\eta)(1-\mu)$ & leakage \\
\hline$(q, \min (e+1, E), \min (a+1, E))$ & $\eta(1-\lambda)(1-\mu)$ & energy arrival \\
\hline \hline \multicolumn{2}{|c|}{ PACKET ARRIVAL, NO SERVICE } & explanation \\
\hline \hline$\rightarrow$ new state & probability & no energy variation \\
\hline$(\min (q+1, Q), e, a)$ & $\lambda(1-\eta)(1-\mu)(1-\beta-\gamma)$ & charge recovery \\
\hline$(\min (q+1, Q), e, \min (a+1, e))$ & $\lambda \beta(1-\eta)(1-\mu)$ & leakage \\
\hline$\left(\min (q+1, Q),[e-1]^{+},[a-1]^{+}\right)$ & $\lambda \gamma(1-\eta)(1-\mu)$ & energy arrival \\
\hline$(\min (q+1, Q), \min (e+1, E), \min (a+1, E))$ & $\lambda \eta(1-\mu)$ & explanation \\
\hline \hline \multicolumn{2}{|c|}{ NO PACKET ARRIVAL, SERVICE } & normal discharge \\
\hline \hline$\rightarrow$ new state & probability & deep discharge \\
\hline$\left([q-1]^{+},[e-1]^{+},[a-1]^{+}\right)$ & $\mu(1-\lambda)(1-\eta)(1-\alpha)$ & energy arrival \\
\hline$\left([q-1]^{+},[e-1]^{+},[\max (a-2, e-\Delta-1)]^{+}\right)$ & $\mu(1-\lambda)(1-\eta) \alpha$ & \\
\hline$\left([q-1]^{+}, e, a\right)$ & $\mu(1-\lambda) \eta$ & explanation \\
\hline \hline \multicolumn{2}{|c|}{ PACKET ARRIVAL, SERVICE } & normal discharge \\
\hline \hline$\rightarrow$ new state & probability & deep discharge \\
\hline$\left(q,[e-1]^{+},[a-1]^{+}\right)$ & $\mu \lambda(1-\eta)(1-\alpha)$ & energy arrival \\
\hline$\left(q,[e-1]^{+},[\max (a-2, e-\Delta-1)]^{+}\right)$ & $\mu \lambda(1-\eta) \alpha$ & \\
\hline$(q, e, a)$ & $\mu \lambda \eta$ &
\end{tabular}

equations that ultimately lead to a steady-state solution of the system. It is straightforward to prove that the resulting chain is positive recurrent. ${ }^{2}$ For the sake of readability, instead of just giving the balance equations, we detail a step-wise derivation of the individual transitions. The next section presents such a constructive derivation of the transition matrix $\mathbf{T}$.

\section{MARKov Model SOlution}

Some preliminary remarks help writing down the chain. Observe that the data queue has three options about what can happen in a time slot: (i) a data packet arrives, and none is served, then $q$ increases by one, if $q<Q$ (otherwise, the packet is discarded and $q$ is still equal to $Q$ ); (ii) a data packet is served (if $q>0$ ), and none arrives, then $q$ decreases by 1 ; (iii) or $q$ is left unchanged, since either nothing arrives to the queue nor packets are served, or a simultaneous service an arrival take place. Note that we do not exclude the case of simultaneous arrival and departure from the queue, even though this event has smaller probability to happen.

Analogously, we can derive the same transitions for the true energy level, only replacing data arrivals with the generation of an energy quantum from the harvesting mechanism. In addition, $e$ is also subject to the consequences of leakage, so we assume that when there is no transmission in a specific slot, still $e$ can decrease by 1 with probability $\gamma$.

Finally, the apparent energy level $a$ can instead increase by one because of two events: either an energy quantum is harvested from the environment (and none is consumed in the same time slot) or, if $a<e$, a charge recovery event can happen. The probability of this event is $\beta$, unless $e=E$, in which case we assumed that charge is recovered with probability $1-\gamma$. Conversely, $a$ can also decrease because of

${ }^{2}$ Data stability $\lambda<\mu$ and energy stability $\eta<\mu$ can be imposed. However, $G e o / G / 1 / K$ queues are blocking systems and admit a steady-state even if those conditions are not met. Yet, in that case the stochastic analysis will have little utility (e.g., energy outages never happen). a packet transmission that is not combined with an energy arrival in the battery. When this happens, the discharge is deep with probability $\alpha$, meaning that the apparent energy level is decremented by 2 . Otherwise, with probability $1-\alpha$, the discharge is normal and $a$ just decreases by 1 . Incidentally, this kind of transition breaks the quasi-birth-and-death model of similar investigations [16], but it is still manageable. Note that leakage affects $a$ in the same way as it does for $e$. The cases where either an energy quantum arrives but at the same time another one is spent, or nothing arrives and no quantum is spent, still lead to a transition where $a$ is unchanged.

Thus, we can consider the transitions reported by Table I and collect them to derive matrix T. However, the table must be properly read in that, whenever to destination states are the same (as happens on border cases, e.g., $q=0$ or $a=e$, the corresponding transition probabilities must be summed. The table has to be adapted for the case $a=0$; in this case, no service is allowed, therefore one should look only at the first two parts ("no service") and also remove any term $(1-\mu)$ from the probability, since the event of no service happens with probability 1 . As a final remark, note that leakage is still possible when $a=0$ but it only affects the true energy level.

The transitions reported by the table can be collected into an $N \times N$ transition matrix $\mathbf{T}$, with the generic element $t_{i j}$ of $\mathbf{T}$ being the transition probability from the $i$ th state to the $j$ th, according to a given exhaustive labeling of the triples $(q, e, a)$. We are actually interested in finding the steadystate probabilities of the Markov chain, which represent the probabilities of finding the system in a given state in stationary conditions. These values can be represented by the $1 \times N$ row vector $\pi$, which is the solution of the fixed point condition $\boldsymbol{\pi}=\boldsymbol{\pi} \mathbf{T}$, combined with a normalization condition $\boldsymbol{\pi} \mathbf{1}^{\mathrm{T}}=1$, where $\mathbf{1}$ is an all-one row vector. The last condition is required because the columns of $\mathbf{T}$ are not linearly independent, yet we can exploit the fact that $\pi$ is a vector of state probabilities.

Vector $\pi$ can be used to derive several metrics of interest. 


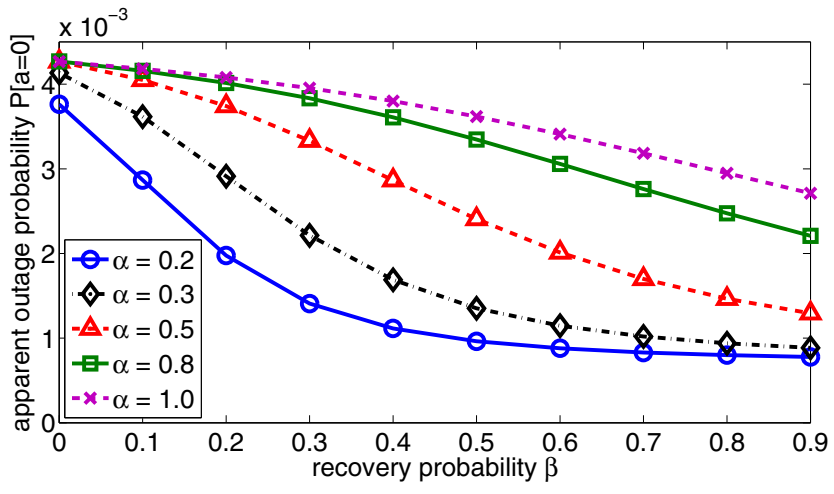

Fig. 1. Apparent outage probability for our scenario.

The steady-state probabilities of certain events (thereafter simply referred to as their "probabilities") can be computed by considering the $\pi$-terms of the corresponding states. It may be useful to focus on the energy outage events [3], describing that the device is no longer powered. Energy outage is a generally undesirable event that can be partly avoided by means of clever transmission policy design. However, two aspects of our expanded model are in special relationship with that event.

Outage is of special interest for our analysis, as opposed to other undesirable events such as energy overflow (i.e., the true level of the battery exceeding $E$, which means that energy is wasted). This is because our model involves leakage effects and deep discharges that can drain the battery even more. Also importantly, we considered two separate energy levels, therefore we expect that multiple definitions of outage coexist. In the next section, we will consider a practical evaluation case and discuss this point even further.

\section{NumericAl RESUlts}

We show quantitative evaluations resulting from the application of the model previously outlined. The device parameters are set as follows. The maximum data queue and energy queue lengths are $Q=20, E=20$, with a maximum gap $\Delta=6$ between the apparent and the true energy levels. This results in 4368 states, as per (1). The leakage rate is $\gamma=0.1$, while $\alpha$ and $\beta$ are kept variable to evaluate their impacts. Arrival rates to the queue are set as $\lambda=0.5$ for the data packets and $\eta=0.6$ for the energy quanta, while the service rate of both queues is set to $\mu=0.7$. Notice that our choices imply stability of all the involved queues; however, $\mu$ is still sufficiently far from an aggressive battery usage that would drain the harvested energy quite soon, thereby causing frequent outages. We tried other scenarios and the result were always found to be in agreement with those shown here.

We evaluate the probabilities of the following events. The real outage event is defined as $[e=0]$ and happens when the device is truly out of charge. If the apparent level $a$ reaches 0 , we are just in apparent outage; note that this does not necessarily implies that the battery is without charge; actually, if $a=0$ but $e>0$, it is possible to "recover" some charge thanks to the recovery effect, even in the absence of energy arrivals. Thus, we finally evaluate the correct discharge notice as $[e=0 \mid a=0]$. All of the metrics are plotted versus the

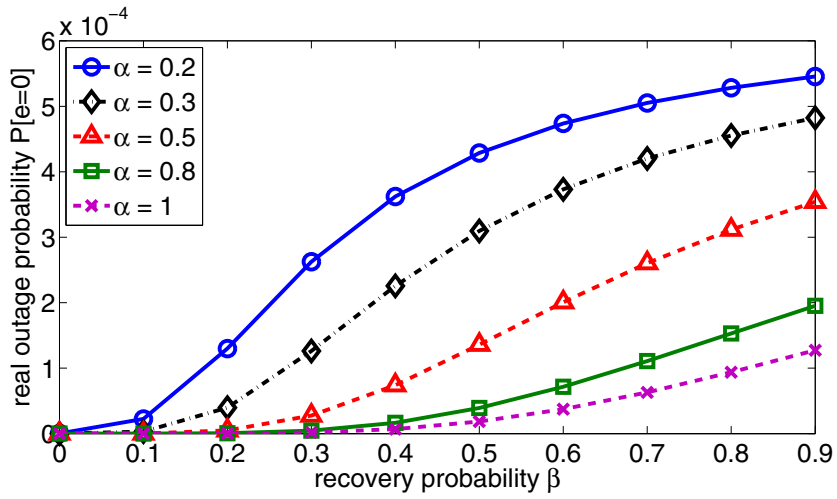

Fig. 2. Real outage probability for our scenario.

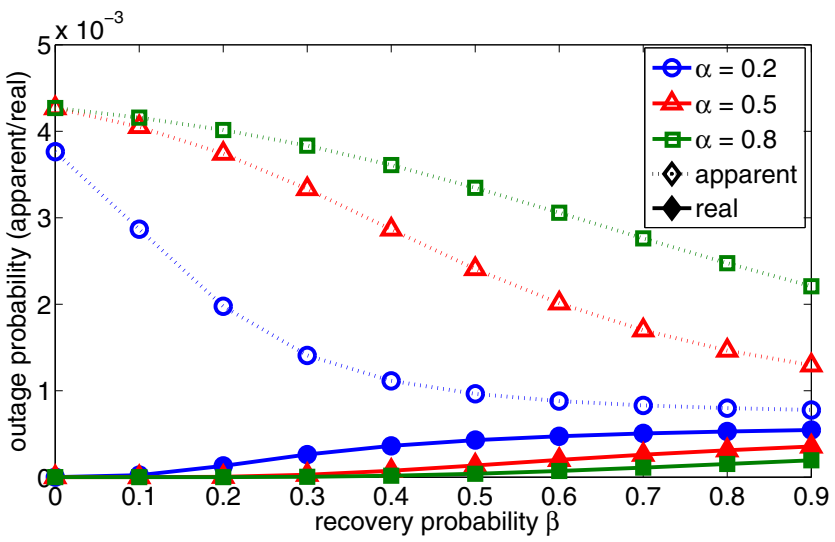

Fig. 3. Comparison between apparent and real outages.

charge recovery parameter $\beta$ (which must be between 0 and $1-\gamma=0.9)$ and for various choices of the deep discharge probability $\alpha$. This means that for low values of $\alpha$ and/or high values of $\beta$ the charge recovery effect more frequently keeps $e$ and $a$ around the same value. Conversely, the larger $\alpha$ and the lower $\beta$, the higher the average gap between the two levels.

Fig. 1 shows the probability of apparent outage. For high values of $\beta$, the curves have a floor to a lower bound. This value is not zero: for our specific numerical choices, it is equal to $7.8 \cdot 10^{-4}$. However, low values of $\beta$ can lead to a much higher apparent outage probability as high as $4.3 \cdot 10^{-3}$, more than five times higher. Moreover, the bound is actually loose if $\alpha$ is high, which means that even a high recovery probability cannot catch up the frequent deep discharges and therefore the apparent outage probability does not decrease significantly.

Fig. 2 shows the real outage probability. It may surprise that the curves increase in $\beta$ and are actually very close to 0 for $\beta \rightarrow 0$. This counterintuitive behavior can be explained by observing that the device stops operating at the apparent outage, and not at the real outage event. As previously shown, this occurs more frequently when $\alpha$ is high and $\beta$ is low, in which cases the real outage event is very rare. However, the results also show that, among the considered cases, the highest real outage probability is equal to $5.5 \cdot 10^{-4}$ for $\alpha=0.2$ and $\beta=0.9$. Thus, even when they are most frequent, real outages do not occur nearly as often as the apparent ones.

Such a comparison between apparent and real outage is summarized by Fig. 3. It is highlighted that the curves ap- 


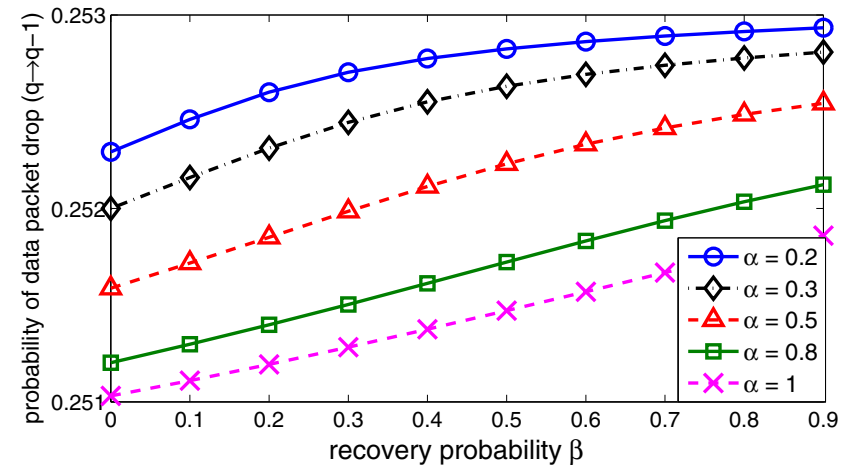

Fig. 4. Probability of packet drop for our scenario.

proach only for low $\alpha$ and high $\beta$. At the right-most end of the curve, $\alpha=0.2$ still leads to more than $25 \%$ of difference. This gap can grow up to one order of magnitude, as if the case, for example, when $\alpha=0.8$ and $\beta=0.3$.

Fig. 4 shows the probability of the packet drop when no packet arrival is observed (transition from $q$ to $q-1$ state). It seems logical, that higher probability of charge recovery leads to lower meaning of apparent outage which increases the probability of data packet drop, because a data packet cannot be transmitted if the battery is depleted $(a=0)$.

Finally, Fig. 5 shows the probability of correct discharge notice. This is the conditional probability that the battery is truly without any charge when the apparent level is 0 . Yet, the figure shows that this is not very likely. Even in the best considered case, there is a $30 \%$ of false alarm when the battery is recognized as apparently discharged. Also, if $\alpha>0.5$ the probability of correct discharge notice is less than $30 \%$ even in the best case of a very high recovery probability $\beta$.

\section{Conclusions}

We developed a stochastic model for battery-powered energy harvesting mobile devices, based on a discrete-time finitestate Markov chain. We keep into account the data queue and the energy level of the device; the latter is mapped through variables $a$ and $e$, representing the apparent and true state of the battery, respectively. This way, we can represent non-idealities such as leakage and more importantly charge recovery, which is generally overlooked in most investigations.

We applied the model to sample evaluations, capturing energy outage under two definitions, i.e., depending on whether $a$ is zero, or $e$ is. The main conclusion is that charge recovery severely affects the performance, as the apparent outage probability can be significantly larger than that of real outage. Conversely, the probability that $e$ actually reaches 0 is lowered by the early operational stop caused by reaching an apparent level $a$ equal to 0 , which can lead to heavy underutilization of the device. The key parameters in determining the extent of this phenomenon are the probabilities of deep discharge and recovery, which should be kept low and high, respectively.

For simplicity, we considered constant parameters in the Markov chain. The main point of our contribution is proven in spite of this simplifying assumptions, although future work may relax this assumption and investigate exponential or

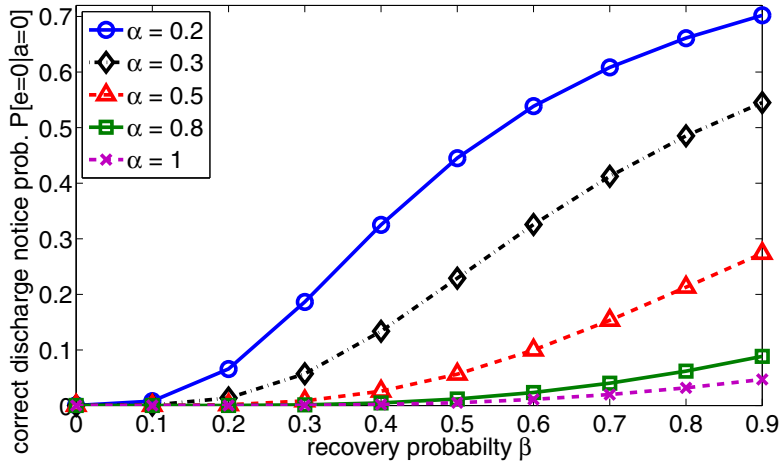

Fig. 5. Probability of correct discharge notice.

multi-step [15] discharging. We can also include a dependence on the energy level in the leakage probability. Finally, we can merge the present contribution with the investigations of [12] about correlation in data and energy arrivals, or those of [3] where the service rate depends on the energy level, to optimize the battery usage under an overall more realistic model.

\section{REFERENCES}

[1] M. P. Mills, "The cloud begins with coal," Digital Power Group, 2013

[2] I. Humar, X. Ge, L. Xiang, M. Jo, M. Chen, and J. Zhang "Rethinking energy efficiency models of cellular networks with embodied energy," IEEE Network, vol. 25, no. 2, pp. 40-49, 2011.

[3] N. Michelusi, L. Badia, and M. Zorzi, "Optimal transmission policies for energy harvesting devices with limited state-of-charge knowledge," IEEE Trans. Commun., vol. 62, no. 11, pp. 3969-3982, 2014.

[4] J. A. Paradiso and T. Starner, "Energy scavenging for mobile and wireless electronics," IEEE Perv. Comput., vol. 4, pp. 18-27, 2005.

[5] M. Gatzianas, L. Georgiadis, and L. Tassiulas, "Control of wireless networks with rechargeable batteries," IEEE Trans. Wirel. Commun., vol. 9, pp. 581-593, 2010.

[6] J. Ventura and K. Chowdhury, "Markov modeling of energy harvesting body sensor networks," Proc. IEEE PiMRC, pp. 2168-2172, 2011.

[7] C. M. Krishna, "Managing battery and supercapacitor resources for realtime sporadic work," IEEE Emb. Sys. Lett., vol. 3, no. 1, pp. 32-36, 2011.

[8] A. Baumgardt, F. Bachheibl, and D. Gerling, "Utilization of the battery recovery effect in hybrid and electric vehicle applications," Proc. 17th Int. Conf. on Elec. Mach. and Syst., pp. 254-260, 2014.

[9] K. Wei, W. Zhang, and Y. Yang, "Prolonging battery usage time in smartphones," Proc. IEEE ICC, 2013.

[10] P. M. Gomadam, J. W. Weidner, R. A. Dougal, R. E. White, "Mathematical modeling of lithium-ion and nickel battery systems," J. Pow. Sources, vol. 110, pp. 267-284, 2002.

[11] R. Rao, S. Vrudhula, and D. Rakhmatov, "Battery modeling for energyaware system design," IEEE Comp.Mag., vol. 36, no. 12,pp. 77-87, 2003.

[12] N. Michelusi, L. Badia, R. Carli, L. Corradini, and M. Zorzi, "Energy management policies for harvesting-based wireless sensor devices with battery degradation," IEEE Trans. Commun., vol. 61, no. 12, pp. 49344947, 2013.

[13] K. Tutuncuoglu, A. Yener, and S. Ulukus, "Optimum policies for an energy harvesting transmitter under energy storage losses," IEEE J. Sel. Areas Commun., vol. 33, no. 3, pp. 467-481, 2015.

[14] B. Devillers and D. Gündüz, "A general framework for the optimization of energy harvesting communication systems with battery imperfections," J. Commun. Netw., vol. 14, no. 2, pp. 130-139, 2012.

[15] C. F. Chiasserini and R. R. Rao, "Energy efficient battery management," IEEE J. Sel. Ar. Commun., vol. 19, no. 7, pp. 1235-1245, 2001.

[16] L. Badia, "On the impact of correlated arrivals and errors on ARQ delay terms," IEEE Trans. Comm., vol. 57, no. 2, pp. 334-338, 2009.

[17] I. Kaj and V. Konané, "Modeling battery cells under discharge using kinetic and stochastic battery models," Applied Mathematical Modelling, vol. 40, no. 17-18, pp. 7901-7915, 2016.

[18] M.D. Levi, D. Aurbach, "Diffusion coefficients of lithium ions during intercalation into graphite derived from the simultaneous measurements and modeling of electrochemical impedance and potentiostatic intermittent titration characteristics of thin graphite electrodes," J. Phys. Chem, vol. 101, no. 23, pp. 4641-4647, 1997. 